\title{
On a Completely Integrable Numerical Scheme for a Nonlinear Shallow-Water Wave Equation
}

\author{
Roberto CAMASSA ${ }^{\dagger}$, Jingfang HUANG ${ }^{\ddagger}$ and Long LEE $~$ \\ Department of Mathematics, University of North Carolina \\ Chapel Hill, NC 27599, USA \\ E-mail: †'camassa@amath.unc.edu, ${ }^{\ddagger} h u a n g @ a m a t h . u n c . e d u,{ }^{\mathbb{I}}$ longlee@amath.unc.edu
}

This article is part of the special issue published in honour of Francesco Calogero on the occasion of his 70th birthday

\begin{abstract}
An algorithm for an asymptotic model of wave propagation in shallow-water is proposed and analyzed. The algorithm is based on the Hamiltonian structure of the equation, and corresponds to a completely integrable particle lattice. Each "particle" in this method travels along a characteristic curve of the shallow water equation. The resulting system of nonlinear ordinary differential equations can have solutions that blow up in finite time. Conditions for global existence are isolated and convergence of the method is proved in the limit of zero spatial step size and infinite number of particles. A fast summation algorithm is introduced to evaluate integrals in the particle method so as to reduce computational cost from $O\left(N^{2}\right)$ to $O(N)$, where $N$ is the number of particles. Accuracy tests based on exact solutions and invariants of motion assess the global properties of the method. Finally, results on the study of the nonlinear equation posed in the quarter (space-time) plane are presented. The minimum number of boundary conditions required for solution uniqueness and the complete integrability are discussed in this case, while a modified particle scheme illustrates the evolution of solutions with numerical examples.
\end{abstract}

\section{Introduction}

The nonlinear partial differential equation (PDE) of evolution

$$
u_{t}+2 \kappa u_{x}-u_{x x t}+3 u u_{x}=2 u_{x} u_{x x}+u u_{x x x}
$$

results from an asymptotic expansion of the Euler equations governing the motion of an inviscid fluid whose free surface can exhibit gravity driven wave propagation $[9,14]$. The small parameters used to carry out the expansion are the aspect ratio, whereby the depth of the fluid is assumed to be much smaller than the typical wavelength of the motion, and the amplitude ratio, or ratio between a typical amplitude of wave motion and the average depth of the fluid. Thus, the equation is a member of the class of weakly nonlinear (due to the smallness assumption on the amplitude parameter) and weakly dispersive (due to the long wave assumption parameter) models for water wave propagation. However, at variance with its celebrated close relatives in this class, such as the Korteweg-de Vries (KdV) and Benjamin-Bona-Mahony (BBM) equations, these small parameters are assumed to be linked only by a relative ordering, rather than a power law relation. This allows 
to retain terms on the right hand side that would be of higher order with respect to both the $\mathrm{KdV}$ and BBM expansions, and, in principle, consider dynamical regimes in which nonlinearity is somewhat dominant with respect to wave dispersion.

The choice of the dimensional form (1.1) is dictated by its relative simplicity. In fact, the equation written in this way refers to a frame of reference moving at uniform speed, which allows to eliminate extra terms arising from the derivation of the equation in the lab frame of reference (where the fluid can be considered at rest at some boundary). Thus, in the form presented here the dependent variable $u$ refers to the horizontal fluid velocity along the $x$-direction as measured at time $t$ by an observer moving at speed $\kappa$. This speed is related to the critical shallow water wave speed $\sqrt{g h_{0}}$, where $g$ is the gravity acceleration and $h_{0}$ is the undisturbed water depth.

The physical foundations of equation (1.1) are accompanied by some peculiar mathematical features. These have received some attention in the recent literature, and no attempt will be made here to provide a detailed reference list. Suffices to say that, like the KdV model, the equation possesses the remarkable property of complete integrability, as evidenced by its Laxpair representation. Moreover, this property is complemented by the existence of a class of weak solutions that can serve as a natural projection of the general solution of (1.1) to an approximating (but still completely integrable) finite dimensional dynamical system [6, 9]. This system of ordinary differential equations (ODEs) can be viewed as describing particles interacting through a long range potential (here position and momentum dependent), which expresses advection of the particles by the velocity $u$ of the shallow water equation (1.1). The velocity is in turn determined by the particle positions and momenta.

The present work focuses on developing and analyzing a numerical scheme based on the above mentioned projection to weak solutions. Our emphasis is on simplicity and efficiency, rather than high-order accuracy, though increased accuracy can be achieved with a modicum of extra effort. In Section 2 we review the integrable formulation for the shallow-water equation (1.1) and show how the particle method arises from this formulation in a straightforward manner. We then establish the properties of the method from the viewpoint of ODEs, and, in particular, show that for a relatively wide class of initial data there are no particle collisions in finite times. This no-collision property is followed by the theory of convergence for the particle method presented in Section 3. Next, Section 4 improves the particle algorithm by implementing a fast summation to reduce the computational cost from $O\left(N^{2}\right)$ to $O(N)$, where $N$ is the number of particles. Sections 5 presents a few tests of the particle method. The tests for the case $\kappa=0$ show evidence of instability, which turns out to be related to clustering of particles, in theory forbidden for finite times but in practice occurring due to the exponentially fast decay of inter-particle distance. A simple redistribution algorithm to prevent instabilities of this kind is then introduced in Section 5.1. Finally, Section 6 ventures briefly into the study of the initial-boundary value problem for the nonlinear evolution equation (1.1).

\section{The integrable formulation}

By introducing the characteristics $x=q(\xi, t)$ emanating from $x=\xi$ at $t=0$,

$$
\frac{d q}{d t}=u(q(\xi, t), t), \quad q(\xi, 0)=\xi,
$$


a solution of equation (1.1) follows formally from the Hamiltonian system

$$
\begin{aligned}
& q_{t}(\xi, t)=\frac{1}{2} \int_{-\infty}^{\infty} e^{-|q(\xi, t)-q(\eta, t)|} p(\eta, t) d \eta-\kappa, \\
& p_{t}(\xi, t)=\frac{1}{2} \int_{-\infty}^{\infty} \operatorname{sgn}(\xi-\eta) e^{-|q(\xi, t)-q(\eta, t)|} p(\xi, t) p(\eta, t) d \eta .
\end{aligned}
$$

Here the characteristics $q(\xi, t)$ play the role of positions conjugate to the momentum-like variables $p(\xi, t)[6]$ in the Hamiltonian

$$
H=\frac{1}{4} \int_{-\infty}^{\infty} \int_{-\infty}^{\infty}\left(e^{-|q(\xi, t)-q(\eta, t)|} p(\eta, t) p(\xi, t)-\kappa(p(\xi, t)+p(\eta, t))\right) d \eta d \xi,
$$

which yields system (2.2) by the (standard) Poisson structure

$$
q_{t}=\frac{\delta H}{\delta p}, \quad p_{t}=-\frac{\delta H}{\delta q},
$$

with functional derivatives $\delta / \delta q, \delta / \delta p$ with respect to the functions $q(\xi, t)$ and $p(\xi, t)$ at fixed time $t$. The choice of initial condition for the position variable, dictated by the characteristics condition, implies $q_{\xi}(\xi, 0)=1$, so that the constraint

$$
q_{\xi}(\xi, t)=\frac{p(\xi, 0)}{p(\xi, t)}
$$

is maintained at all times of existence of the solution $(q(\xi, t), p(\xi, t))$. Thus, the momentum variable $p(\xi, t)$ could be eliminated from the system to obtain an evolution equation containing only the dependent variable $q(\xi, t)$ and its first derivative with respect to the initial label $\xi$. Vanishing of this derivative generically corresponds to crossing of characteristics curves, with loss of uniqueness of solutions $\xi(x, \cdot)$ to the equation $x=q(\xi, \cdot)$. Constraint (2.4) implies that if the initial condition $p(\xi, 0)$ does not have zeros, then $q_{\xi}(\cdot, t)$ is bounded away from zero, thereby preventing characteristics from crossing, for as long as $|p(\cdot, t)|<\infty[6]$.

The relation of system (2.2) with the original form (1.1) of the shallow water equation results from the definition of the velocity $u(x, t)$ in terms of characteristics $q(\xi, t)$ and the conjugate momentum $p(\xi, t)$,

$$
u(x, t)=-\kappa+\frac{1}{2} \int_{-\infty}^{\infty} e^{-|x-q(\eta, t)|} p(\eta, t) d \eta .
$$

Equation (1.1) assumes a particularly compact form [9] by defining the auxiliary field $m(x, t)$ as $m(x, t) \equiv\left(1-\partial_{x}^{2}\right) u(x, t)$. Replacing $u-u_{x x}$ by the auxiliary $m$, equation (1.1) can be written as

$$
m_{t}+u m_{x}=-2(m+\kappa) u_{x} .
$$

Substituting (2.5) into $m$, we obtain

$$
m(\xi, t)=-\kappa+\frac{p(\xi, t)}{q_{\xi}(\xi, t)},
$$

Notice that the initial condition $q_{\xi}(\xi, 0)=1$ and relation (2.7) imply $m_{0}(\xi)+\kappa=p(\xi, 0)$, where $m_{0}(\cdot)$ is the initial value of $m(\cdot, t)$. Hence the constraint $(2.4)$ can also read $[5,6,12]$

$$
p(\xi, t)=\frac{m_{0}(\xi)+\kappa}{q_{\xi}(\xi, t)} .
$$


For the numerical algorithm to be developed later, it is convenient to put system (2.2) in a slightly different (but entirely equivalent) form,

$$
\begin{aligned}
& q_{t}(\xi, t)=\frac{1}{2} \int_{-\infty}^{\infty} e^{-|q(\xi, t)-q(\eta, t)|}\left(p(\eta, t)-\kappa \frac{\partial q}{\partial \eta}(\eta, t)\right) d \eta, \\
& p_{t}(\xi, t)=\frac{1}{2} p(\xi, t) \int_{-\infty}^{\infty} \operatorname{sgn}(\xi-\eta) e^{-|q(\xi, t)-q(\eta, t)|}\left(p(\eta, t)-\kappa \frac{\partial q}{\partial \eta}(\eta, t)\right) d \eta,
\end{aligned}
$$

where we have used the properties of the kernel exp $|x-y|$ to take the constant $\kappa$ under the integral sign.

\section{Discrete integrable system}

The numerical algorithm proposed in [6] approximates the integrals in equation (2.2) by their Riemann sums, thereby yielding Hamiltonian systems for "particles" with coordinates

$$
q_{i}(t) \equiv q\left(\xi_{i}, t\right)
$$

and momenta

$$
p_{i}(t) \equiv p\left(\xi_{i}, t\right),
$$

where $\xi_{i}=\Xi+i h$ for some real $\Xi$, step-size $h>0$ and $i=1, \cdots, N$.

If we replace $q_{\eta}$ in the integrand of equation $(2.9 \mathrm{a}, 2.9 \mathrm{~b})$ by formula $(2.4)$, then the finite dimensional system of ODEs for $N$ particles for equation (2.9) can be written as

$$
\begin{aligned}
& \dot{q}_{i}=\frac{h}{2} \sum_{j=1}^{N} e^{-\left|q_{i}-q_{j}\right|} p_{j}-\frac{h}{2} \kappa \sum_{j=1}^{N} e^{-\left|q_{i}-q_{j}\right|} \frac{p_{j}^{0}}{p_{j}}, \\
& \dot{p}_{i}=\frac{h}{2} p_{i} \sum_{i \neq j=1}^{N} \operatorname{sgn}\left(q_{i}-q_{j}\right) e^{-\left|q_{i}-q j\right|} p_{j}-\frac{h}{2} \kappa p_{i} \sum_{i \neq j=1}^{N} \operatorname{sgn}\left(q_{i}-q_{j}\right) e^{-\left|q_{i}-q_{j}\right|} \frac{p_{j}^{0}}{p_{j}},
\end{aligned}
$$

where $p\left(\xi_{j}, 0\right) \equiv p_{j}^{0}$. We will refer to system (3.1) as the particle method for solving the shallow-water equation (1.1).

We remark that particle methods have been discussed in the context of evolution equations with similar structure in [10], and, while different from ours, the approach used therein can result in a similar system of ODEs.

It is also worth pointing out that the $(q, p)$ system is in principle more general than the shallow water equation (1.1), which follows from system (2.2) as a particular case once the constraint (2.4) is taken into account. The constraint has the effect of removing one "degree of freedom" from the system, by effectively projecting it to an invariant solution manifold where the dynamics is governed by equation (1.1) where only the first time-derivative appears.

It is remarkable that system (2.2) itself follows from a more general class of systems which possess a Lax-pair, and hence it belongs to the family of completely integrable equations. It can be shown [6] that the isospectral problem

$$
\lambda \phi(\xi, t)=\int_{-\infty}^{\infty} k(\xi, \eta ; t) \phi(\eta, t) d \eta, \quad \phi_{t}(\xi, t)=\frac{1}{2} \int_{-\infty}^{\infty} \operatorname{sgn}(\xi-\eta) k(\xi, \eta ; t) \phi(\eta, t) d \eta,
$$


yields system (2.2) for the special choice of the symmetric kernel $k(\xi, \eta ; t)$ given in terms of $q(\xi, t)$ and (non-negative) $p(\xi, t)$ by

$$
k(\xi, \eta ; t)=\frac{1}{2} e^{-\frac{1}{2}|q(\xi, t)-q(\eta, t)|} \sqrt{p(\xi, t) p(\eta, t)} .
$$

The same Riemann sums approximation to integrals used to derive the particle systems now yields its Lax pair through the matrix approximation to the kernel $k\left(\xi_{i}, \eta_{j} ; t\right) \equiv h k_{i j}(t)$,

$$
\lambda \phi_{i}(t)=\sum_{j=1}^{N} k_{i j}(t) \phi_{j}(t), \quad \dot{\phi}_{i}(t)=\left(\sum_{j=i+1}^{N}-\sum_{j=1}^{i-1}\right) k_{i j}(t) \phi_{j}(t) .
$$

The numerical scheme based on the particle method relies on existence and uniqueness of solutions of the initial value problem for system (3.1). Moreover, collisions among particles are undesirable, and it might be expected that these correspond to finite-time singularities in the ODEs' solution.

For simplicity, let us consider the initial value problem for case $\kappa=0$ of the Hamiltonian system (3.1)

$$
\dot{q}_{i}=\frac{1}{2} h \sum_{j=1}^{N} e^{-\left|q_{i}-q_{j}\right|} p_{j}, \quad \dot{p}_{i}=\frac{1}{2} h \sum_{i \neq j=1}^{N} \operatorname{sgn}\left(q_{i}-q_{j}\right) e^{-\left|q_{i}-q_{j}\right|} p_{i} p_{j}
$$

The above discrete system is integrable and possesses an important no-collision principle, namely, if the initial momenta are positive, $p_{i} \geq \epsilon>0, i=1, \ldots N$ for some constant $\epsilon$, then the solution of system (3.5) exists uniquely for all times. In particular, no two particles can occupy the same position $q_{i}(t)=q_{j}(t)$, for some $i \neq j$, at any finite time $t[7]$.

The global existence result can be easily modified to include the case $\kappa \neq 0$, as it is evident from the integrable formulation (2.2), which shows that the $p$-components are simply shifted by the constant $\kappa$. In this case the restriction to positive initial momenta corresponds to $m_{0}(x)+\kappa>0$ by definition (2.8), which has a natural physical interpretation within the asymptotic derivation of the shallow water model (1.1) (Camassa, Huang and Lee, in preparation). Also worth noticing is that while collisions lead to the blow-up of the solution in the momentum components, it is possible to continue the solution past blow-up time(s) by defining an appropriate set of rules, such as energy conservation, using the dynamical system structure of the ODEs [9].

For fixed $h$, the system of ODE (3.5) and its more general form (3.1) are members of the (extended) Toda-lattice family $[15,16,17]$. This connection becomes particularly transparent when viewed from the Lax-pair [6], but, as mentioned above, the complete integrability of the system needs further study in order to be used for addressing issues of practical relevance for the continuum (PDE) solutions of (1.1).

Let $q(\xi, t), p(\xi, t)$ denote the solution to the continuous problem (2.9) corresponding to the initial data

$$
q(\xi, 0)=\xi, \quad p(\xi, 0)=p^{0}(\xi)
$$

while $\tilde{q}(t), \tilde{p}(t)$ will stand for the solution of the particle system (3.5) emanating from initial conditions

$$
\tilde{q}_{i}(0)=q\left(\xi_{i}, 0\right) \equiv \xi_{i}, \quad \tilde{p}_{i}(0)=p^{0}\left(\xi_{i}\right) .
$$


Let $q_{i}(t)=q\left(\xi_{i}, t\right)$ denote the PDE solution evaluated at the grid points, and define the difference between PDE and ODE variables as

$$
\phi_{i}=q_{i}-\tilde{q}_{i}, \quad \psi_{i}=p_{i}-\tilde{p}_{i} .
$$

In what follows, the (discrete) $l_{1}$-norm, is defined by $\|\phi\|=h \sum_{j}\left|\phi_{j}\right|$.

Let the initial total momentum be $P=h / 2 \sum_{i=1}^{N} p_{i}<\infty$. Suppose that (3.6) and (3.7) are the initial data for the system (2.9) and (3.5), respectively, with $\kappa=0$. One can show [7] if the function $p^{0}(\xi)>0$ is sufficiently smooth and decays rapidly at infinity, for any finite time $T>0$ there exists a grid-step size $h$ such that the difference between the continuum and discrete variable satisfies

$$
\|\phi\|+\frac{1}{P}\|\psi\| \leq h^{2} \frac{C}{2 L P}\left(e^{2 L P t}-1\right)
$$

for $0 \leq t<T$, where $C$ is a constant independent of $T$ or $h$ and $L>(1+h / 2) / 2$. Thus, we have established by this estimate a convergence theorem which guarantees a quadratic convergent rate of the particle algorithm to the solutions of the PDE (1.1).

\section{Fast summation algorithm}

The major computational cost for solving the system of $2 N$ equations (3.1) is the cost of evaluating the Riemann sum at each time step. Computing the summations alone takes $O\left(N^{2}\right)$ operations for an $N$-particle system, if no recursion formulas are used.

With the aim of reducing the computational cost for the algorithm, we propose a recursion formula based on the principle of absence of particle collisions in any finite times. This principle allows us to strip the absolute value notation in the power of the exponential function, which in turn makes a recursion relation for evaluating the sums possible. With the help of this recursion formula, the total operations needed for performing the summation is reduced to $O(N)$ for the $N$-particle system. We present the fast summation algorithm for the non-dispersive case $\kappa=0$. The case $\kappa \neq 0$ is completely analogous but leads to longer expressions.

By the no-collision property, the particle method (3.1) has $q_{i}>q_{j}$ if $i>j$ and vice-versa if $i<j$. Hence equation (3.1) can be written as

$$
\begin{aligned}
& \dot{q}_{i}=\frac{h}{2}\left(\sum_{j=1}^{i-1} e^{-\left(q_{i}-q_{j}\right)} p_{j}+p_{i}+\sum_{j=i+1}^{N} e^{-\left(q_{j}-q_{i}\right)} p_{j}\right), \\
& \dot{p}_{i}=\frac{h}{2} p_{i}\left(\sum_{j=1}^{i-1} e^{-\left(q_{i}-q_{j}\right)} p_{j}-\sum_{j=i+1}^{N} e^{-\left(q_{j}-q_{i}\right)} p_{j}\right) .
\end{aligned}
$$

Define new variables:

$$
f_{i}^{l}=\sum_{j=1}^{i-1} e^{-\left(q_{i}-q_{j}\right)} p_{j}, \quad f_{i}^{r}=\sum_{j=i+1}^{N} e^{-\left(q_{j}-q_{i}\right)} p_{j} .
$$

Equation (4.1) then becomes

$$
\dot{q}_{i}=\frac{h}{2}\left(f_{i}^{l}+p_{i}+f_{i}^{r}\right), \quad \dot{p}_{i}=\frac{h}{2} p_{i}\left(f_{i}^{l}-f_{i}^{r}\right) .
$$




\begin{tabular}{|c|c|c|c|c|c|}
\hline$h$ & 0.1 & 0.05 & 0.025 & 0.0125 & 0.00625 \\
\hline$\left\|u-u_{\text {exact }}\right\|$ & $6.90 \mathrm{e}-4$ & $1.94 \mathrm{e}-4$ & $4.65 \mathrm{e}-5$ & $1.17 \mathrm{e}-5$ & $3.03 \mathrm{e}-6$ \\
\hline rate & & 1.83 & 2.06 & 1.99 & 1.93 \\
\hline
\end{tabular}

Table 5.1. Convergence rate for the particle method.

One can see that with a pre-computed $f^{l}$ and $f^{r}$ the number of operations needed for the Riemann sum is $O(N)$ for the system of equations. Since the operations required for $f^{l}$ or $f^{r}$ are also growing as $O(N)$, the total number of operations is $O(N)$. The quantities $f^{l}$ and $f^{r}$ can be solved via the recursion relations

$$
f_{i+1}^{l}=e^{-\left(q_{i+1}-q_{i}\right)}\left(f_{i}^{l}+p_{i}\right) \quad f_{i+1}^{r}=e^{-\left(q_{i}-q_{i+1}\right)} f_{i}^{r}-p_{i+1},
$$

where, because $e^{-\left(q_{i}-q_{i+1}\right)}$ leads to exponential growth, for numerical stability the recursion relation for $f^{r}$ is better solved backward as $f_{i}^{r}=\left(f_{i+1}^{r}+p_{i+1}\right) e^{-\left(q_{i+1}-q_{i}\right)}$.

\section{Numerical results}

This section presents two examples of application of the particle method to the numerical solution of equation (1.1), and examines the accuracy of the scheme via several error estimate criteria. The first example tests the method on a traveling wave solution for the dispersive case $\kappa \neq 0$. The other example deals with initial value problem of the non-dispersive case, $\kappa=0$. The aim is to illustrate the efficiency and the order of accuracy for the scheme. For both cases it may be expected that the solution evolves free of singularities for all times (a priori bounds on the initial condition [9] which ensure that a vertical slope is achieved in finite time at inflection points are not satisfied for the initial conditions we consider). Nevertheless, for the dispersionless case $\kappa=0$ the numerical simulation shows that a rather sharply peaked solitary wave forms and moves away from the origin. From the viewpoint of the particle method, the peaked solution arises from particles clustering rapidly in the region of the peak of the solitary wave(s). Such clustering behavior causes problems in the particle method, as the ordering of the particles becomes affected by numerical errors. An efficient implementation is introduced in this section to remove this complication.

Example 1: Smooth traveling wave solution $\kappa \neq 0$. An explicit exact solution of equation (1.1) is $u(x, t)=U(x-c t) \equiv U(s)[8]$, where $c=8 \kappa / 3$ and $U(s)$ is given by

$$
U(s)=\frac{8}{3} \kappa\left(1-\frac{3 \sqrt{3}+6 \sin 2 z}{(1+2 \cos 2 z)(2 \sqrt{3} \cos 2 z-\sqrt{3} \cos 4 z+2 \sin 2 z+\sin 4 z)}\right),
$$

with $z=\arctan \left(e^{s / 2}\right) / 3$. The initial condition $u_{0}(x)=U(x)$ yields the initial data for the particle algorithm $q(x, 0)=x$ and $p(x, 0)=\kappa+m_{0}(x)$, where

$$
m_{0}(x)=\kappa\left(\frac{c^{2}}{(c-U(x))^{2}}-1\right) .
$$

For our numerical simulation of the traveling wave solution (5.1), we choose $\kappa=1$. All calculations in this section use a fixed ratio $\Delta t / h=1 / 2$, where $\Delta t$ is the time step and $h$ is the spatial grid size. Time integration is performed by an explicit fourth-order Runge-Kutta method. We introduce the (finite) $l_{2}$-norm $\|e\|^{2}=h \sum_{j} e_{j}^{2}$, to study the numerical error between the exact and computed solutions. On a fixed domain [-30,30], at time $t=1$, we list the error in Table 5.1. As the table shows, in this case the particle method is second-order accurate in space, consistently with the 
theory. Next, we consider a wider domain, $[-50,50]$. The traveling wave solution is computed up to time $t=5$. We compare the elapsed CPU time between the fast summation algorithm and the original algorithm with different number of particles. This results in Table 5.2, which

\begin{tabular}{|c|c|c|c|c|c|}
\hline seconds $\backslash N$ & 1000 & 2000 & 4000 & 8000 & 16000 \\
\hline fast summation & 1 & 2 & 4 & 9 & 19 \\
\hline ratio & & 2 & 2 & 2.2 & 2.1 \\
\hline original & 17 & 74 & 290 & 1157 & 4505 \\
\hline ratio & & 4.35 & 3.92 & 3.99 & 3.89 \\
\hline
\end{tabular}

Table 5.2. CPU time for the two summation algorithms. The table shows that the fast summation algorithm is $O(N)$, and the straightforward algorithm is $O\left(N^{2}\right)$, where $N$ is the number of particles.

shows explicitly that the fast summation algorithm for $N$ particles is $O(N)$, while the original straightforward algorithm is $O\left(N^{2}\right)$.

Example 2: Sharp peaked traveling waves for $\kappa=0$ and numerical particle collisions. In ref. [6] during the evolution of the solution $u(x, t)$ out of the initial condition $u(x, 0)$ corresponding to $m_{0}(x)=a \operatorname{sech}^{2}(x)$ (equivalent to $q(\xi, 0)=\xi$ and $p(\xi, 0)=a \operatorname{sech}^{2}(\xi)$ for the particle method), a rather sharply peaked wave forms and moves to the right, followed by others emerging from the location of the initial hump $m_{0}(x)$. During the simulation, one can observe that the particles rapidly cluster in the region of the peak of the solitary waves. Such pile-up phenomenon suggests that particles get very close to each other in this region. When the distance between particles is so close that the machine precision can no longer distinguish between locations of the coalescing particles, particle collisions occur numerically. This effect is of course purely numerical, as we have mentioned in Section 3 that particle collisions cannot take place in finite time. As a consequence of such a numerical artifact, the particle method breaks down shortly after the numerical collision occurs. The first row in Table 5.3 shows the times when numerical collisions occur in the course of computing $p$ and $q$, with respect to increasing floating point precision. The table shows that higher precision arithmetic extends the time for first occurrence of particle collisions, thereby providing evidence that the numerical collision is dominated by the round-off error.

\subsection{Redistribution algorithm}

Taking advantage of the concentration of particles leading numerically to artificial collisions allows to implement a redistribution algorithm rather efficiently: when two particles, with positions $q_{i}$ and $q_{i+1}$, are too close to be distinguished within machine precision, we replace them with one particle at the same location carrying a momentum equal to the sum of $p_{i}$ and $p_{i+1}$. After we carry out this replacement, we relabel the rest of the particles from the original $i+2, \ldots, N$ to $i+1, \ldots, N-1$. In other words, we reduce the dimension of the system of ODEs from $2 N$ to $2 N-2$ by combining any two clustering particles. Of course, this method is somewhat crude in that the moving (Lagrangian) grid can become too sparse in regions away from the clustering particle, as this process of replacement depletes the total number of particles. While it is not too difficult to implement a true redistribution that conserves particles, we leave this out for simplicity.

As we indicate in the first row of Table 5.3, the numerical collision occurs at time $t=54.9$ for the single precision calculation, and at $t=140.3$ for the double precision calculation. There are no numerical collisions for the quadruple precision calculation until $t=317.1$. We perform a calculation up to $t=150$ at each of the three different precisions. The result from the quadruple precision calculation is used as a relative exact solution, since there are no numerical collisions 


\begin{tabular}{|c|c|c|c|}
\hline & single precision & double precision & quadruple precision \\
\hline time & 54.9 & 140.3 & 317.1 \\
\hline $\mathrm{u}(\mathrm{x}=50.6, \mathrm{t}=150)$ & 0.317747861146927 & 0.317904674362106 & 0.317904674362127 \\
\hline
\end{tabular}

Table 5.3. First collision times vs. arithmetic precision of numerics. The second row shows the numerical value of $u$ at the first soliton peak location $x=50.6$ and $t=150$. The redistribution algorithm is employed with the single and double precision arithmetic runs.

(a)

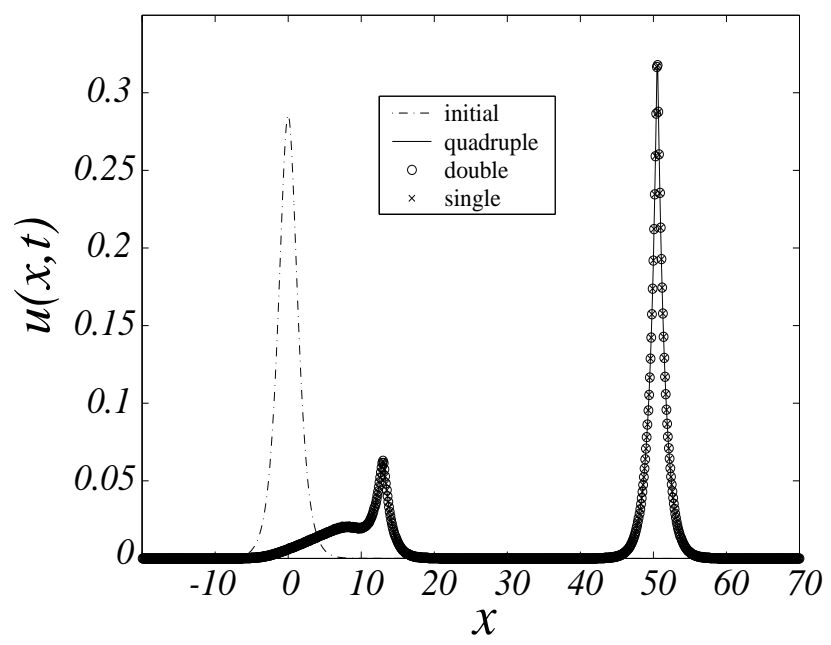

(b)

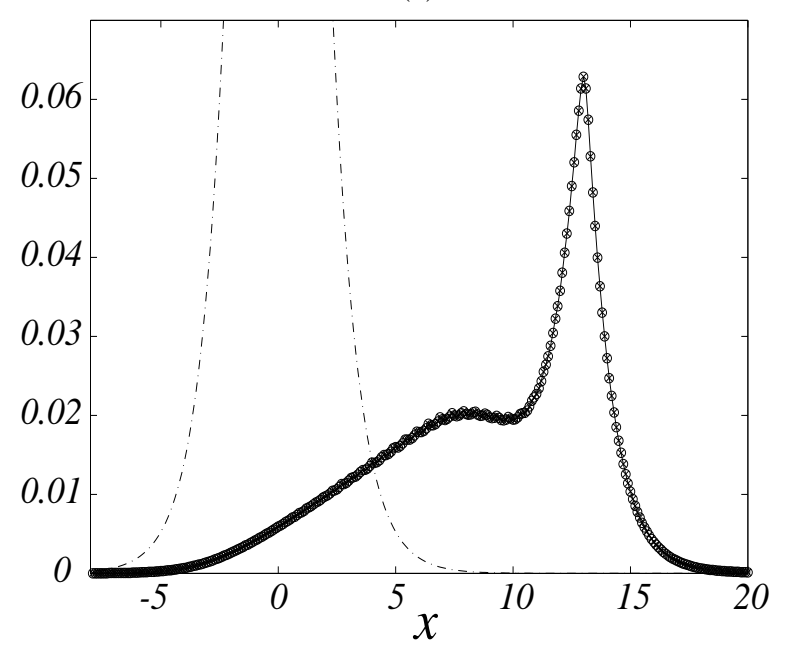

Figure 5.1. (a) Evolution under equation (1.1) for $\kappa=0$ of the initial condition (dash) $m_{0}(x)=a \operatorname{sech}^{2}(x)$, with $a=1 / 2$ for three different precisions, at time $t=150$. (b) Magnification of the region around the second emerging peaked solitary wave.

for this calculation at $t=150$. After applying the redistribution algorithm for the single and the double precision calculation, the second row of Table 5.3 is the computed solution for $u$ at $x=50.6$ where the first peaked soliton is located. These values in the table show that the numerical solution obtained by using the double precision arithmetic matches the relative exact solution up to thirteen digits. The solution obtained by using the single precision arithmetic, however, has only three matching digits. It seems reasonable to attribute this discrepancy to the nonlinearity of the equation, which can amplify the round-off error and the error caused by the redistribution algorithm, since they are both influenced by the different floating-point precision used. Thus, while the same algorithm is used in the two computations, the overall errors for the numerical solutions grow nonlinearly while approaching the near-singular clustering episodes.

Figure 5.1 is the plot of reconstructed solutions at $t=150$ for the three different precisions. The solid line is the result from the quadruple precision. The comparison shows that solutions computed from the redistribution algorithm are indistinguishable from the reference high arithmetic precision solution.

\subsection{Error analysis and the isospectral problem}

As indicated in $\S 2$, the field $p(\xi, t)$ is tied to $q(\xi, t)$ by the constraint $(2.4)$. For the discretization of system (2.9) offered by the particle method, this exact relation between the $p$ and $q$ variables does not survive. However, convergence of the particle method to the PDE solution and its smoothness, 
inherited from appropriate classes of initial data, assures that given a certain time $T$ there exists a step-size $h$ such that (abusing notation a little)

$$
p\left(\xi_{i}, t\right)=p_{i}(t)+O\left(h^{2}\right) \quad \text { and } \quad p\left(\xi_{i}, t\right)=\frac{2 h p_{i}^{0}}{q_{i+1}(t)-q_{i-1}(t)+O\left(h^{3}\right)},
$$

for all times $0<t<T$.

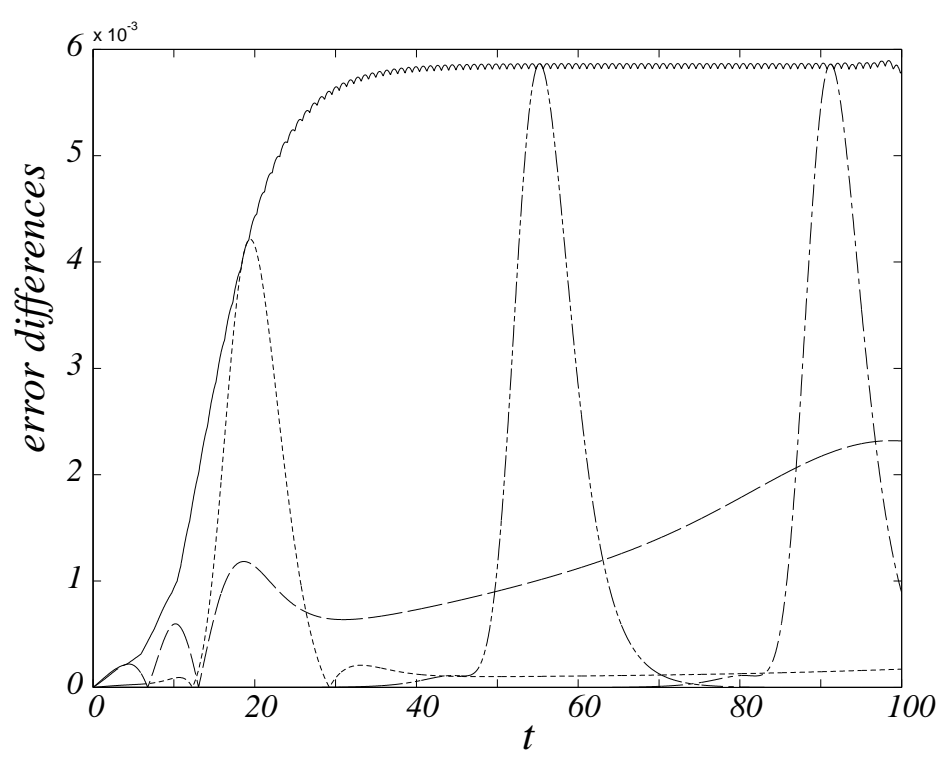

Figure 5.2. Evolution of error differences for the initial value problem of equation $(1.1)$ with $u(x, 0)=$ $1 / 2 \operatorname{sech}^{2}(x), N=8000, h=0.1, \Delta t=0.05$, and $\kappa=0$. Solid curve: error monitor $e_{h}(t)$. Dashed curves: individual particle error $\left|p_{i}(t)-2 h p_{i}^{0} /\left(q_{i+1}(t)-q_{i-1}(t)\right)\right|$, with $i=10$ (long-dashed), $i=20$ (short-dashed), $i=60$ (long-short-short-dashed), $i=100$ (long-short-dashed).

Because the sharpness of the convergence estimates (3.9) is not known precisely, the practical question of how long the solution of the particle method can be trusted to stay close to that of the PDE for a given $h$ is not addressed by the convergence proof. The constraint (2.4) in its version (5.3) offers a different and more systematic way to monitor the error. In fact, apart from spurious cancellations, relations (5.3) show that the deviation

$$
e_{h}(t) \equiv \sup _{i}\left|p_{i}(t)-\frac{2 h p_{i}^{0}}{q_{i+1}(t)-q_{i-1}(t)}\right|
$$

keeps track of the error between the exact PDE solution and its approximation by the particle method. We show this error monitor applied to the general evolution out of an initial hump for $\kappa=0$, for an initial value where an exact solution is not available. Figure 5.2 shows that after an initial transient the error $e_{h}(t)$ grows nonlinearly to saturate at some (mean) constant level. This behavior is related to the evolution of the difference between $p$ 's in the definition (5.3) for each individual particle, when the first peaked wave of Figure 5.1 forms and moves towards the right. This can be seen in the dashed curves of the figure, where this difference is plotted vs. time. Notice that for locations sufficiently far from the origin where the initial hump is located, viz. $i=60$ and $i=100$ in the figure, the $p_{i}(t)$ 's computed by both expressions in (5.3) relax back to zero after the passage of the first wave, and hence so does the error. Also notice that for these particles the individual error suffers a sharp order-of-magnitude increase after an initial slow growth at about 
the times when the first wave reaches these particles' locations. This behavior is related to the clustering effect which we examine in more detail next.

Another, more specific, error monitor can be based on the complete integrability of equation (1.1). If the discrete approximation has to faithfully represent the continuum evolution, the spectrum of the continuum system (3.2) and its discrete counterpart (3.4) need to be close, and the time advancing scheme must leave the spectrum invariant. (Monitoring the spectrum is equivalent to monitoring the integrals of the motion of the shallow water equation through the formulae connecting it to the iterated kernels [6].) This is illustrated by Figure 5.3, where the initial spectrum from the continuum system (3.2), the initial $t=0$ spectrum of (3.4) and its snapshot at time $t=50$ are plotted for the first significant 50 eigenvalues. Here we have taken $\kappa=0, m_{0}(x)=\operatorname{sech}^{2}(x) / 2$, which leads to closed form solution for the (infinite, purely discrete) spectrum of the continuum problem (3.2) [6] $\lambda_{n}=1 /\left(4 n^{2}-1\right)$, for $n=1,2, \ldots$. As one can see, the discretized spectrum drifts away from its continuum counterpart after the first $O\left(N^{1 / 2}\right)$ largest eigenvalues, which gives an indication of the accuracy of the discretization, while the spectrum of the particle scheme remains constant in time (at least within plotting accuracy) for these eigenvalues.

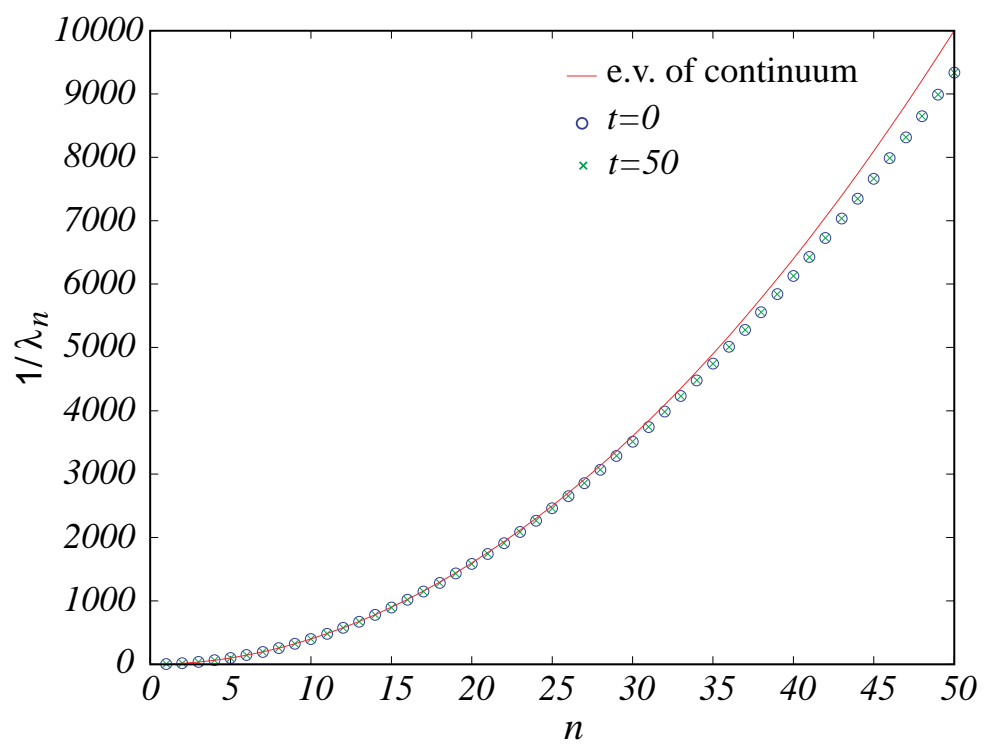

Figure 5.3. Comparison of the first 50 eigenvalues between the continuum problem (3.2) and the discrete system (3.4) with initial condition $m(x, 0)=1 / 2 \operatorname{sech}^{2}(x), N=4000, h=0.025, \Delta t=0.0125$, and $\kappa=0$.

\subsection{A transient phenomenon}

As we have seen in the numerical example for $\kappa=0$, the formation of the sharp (corner-like) peaks shown in Figure 5.1 is associated with the particle clustering discussed in $\S 5$. The long-time asymptotic of the corresponding discrete system studied in [6], however, predicts that each particle in the discrete system eventually breaks out of the cluster and travels alone on a constant speed trajectory, when $t \rightarrow \infty$. For a finite number of particles, it is hence evident that the phenomenon of clustering is merely transient. Fully understanding this transient behavior of the discrete system poses an interesting challenge for the analysis of the PDE (1.1).

With the aim of probing the transient behavior of the discrete system, we revisit the process of formation of clusters by plotting the trajectories of particles in the $(x, t)$ plane for a rather coarse grid. The clustering process acts as a high particle-density front sweeping through the 
computational domain. This front corresponds to the first peaked solitary wave of Figure 5.1. The trailing edge of this quasi-shock wave leaves behind a rarefaction wave. Particles in the rarefaction wave region interact again and form the next clustering of particles, corresponding to the next peaked wave of Figure 5.1; the onset of this second clustering can be seen at the final time in Figure 5.4(a). (Notice that this rarefied area can increase errors due to lack of resolution, especially when coupled with a redistribution algorithm that depletes the total number of particles.)

Figure 5.4(b) shows that the momentum of the front at $t \approx 15$ is completely transferred to the last particle around $t=110$; thereafter this particle travels alone with linearly increasing distance from the rest of the particles. This behavior can be described by the long time asymptotics based on the completely integrable structure of the particle system [6].

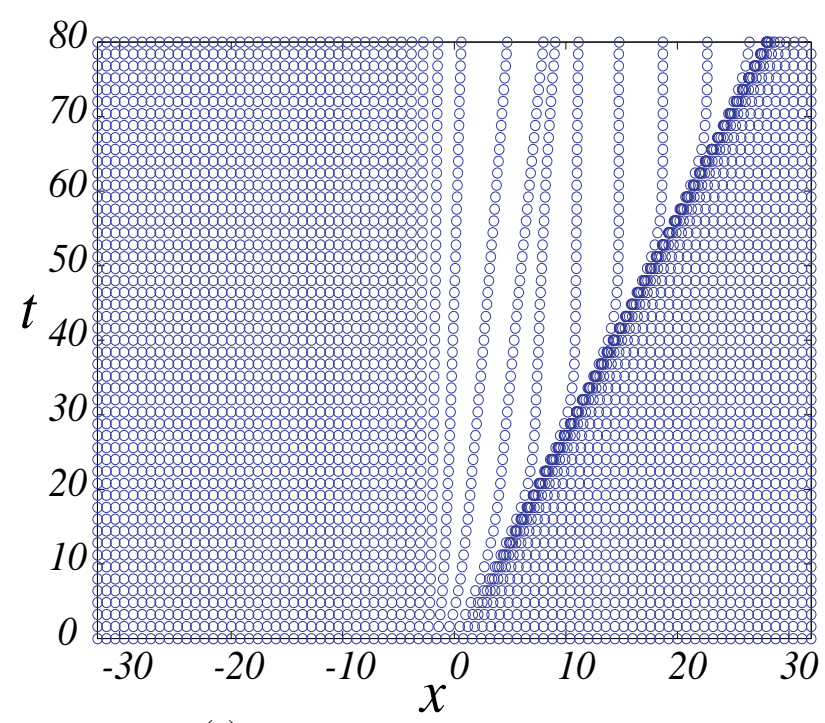

(a)

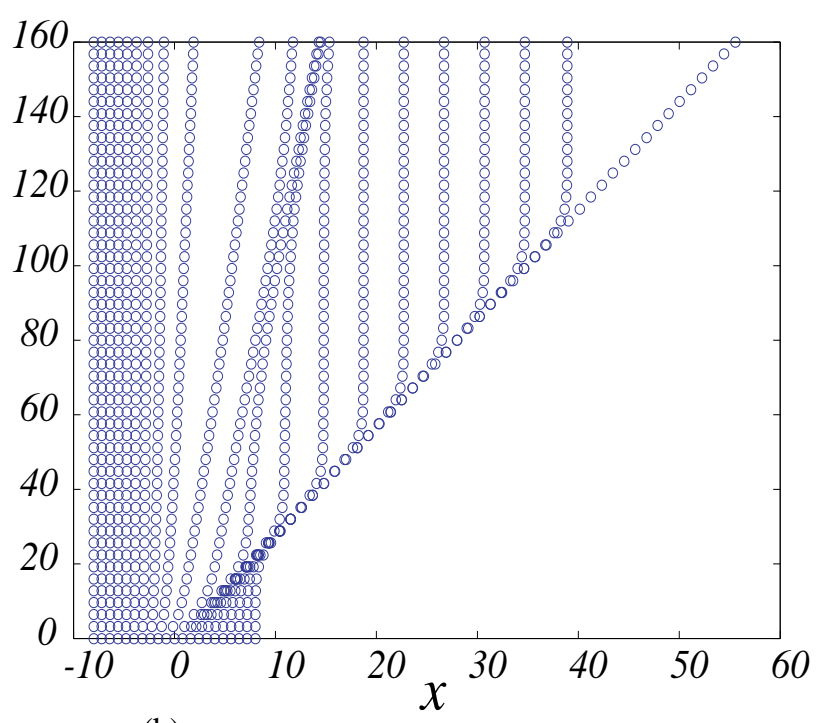

(b)

Figure 5.4. (a) Particle trajectories on the $(x, t)$ plane for the particle method. Here $h=0.8, N=81$ and the final time is $t=80$. The shock-like wave of high particle-density sweeping through the domain at speed $\sim 1 / 3$ can be clearly seen, as well as the next incipient collision near $x=10$. (b) Same as (a) with fewer particles $N=21$; the momentum of the front seen at $t \simeq 15$ is completely transferred to the last (21st) particle around $t=110$. Also seen in this plot is the formation of the second front corresponding to the new emerging peaked wave at $t \simeq 130$.

\section{An initial-boundary value problem}

The "quarter-plane problem" for equation (1.1) is obtained by restricting $x$ to the positive semiaxis and assuming boundary conditions at $x=0$ and for $x \rightarrow \infty$, besides the initial condition $u(x, 0)=f(x)$. For physical applications, it is natural to impose $u(x, t) \rightarrow 0$ as $x \rightarrow \infty$ together with any number of derivatives for any finite time $t$. At $x=0$ the situation is more delicate: it is not immediately obvious how many boundary conditions one must assume without overdetermining the problem. A complete discussion of the initial-boundary value problem is beyond the scope of this paper. In this section, we will only show that assigning the boundary condition $u(0, t)=g(t)$, where $g(t)$ is some smooth function of time with $g(t) \leq 0$ for all $t$, is enough to guarantee uniqueness of the solution. Clearly, this must also constitute a minimal set of boundary conditions. As usual, only the special case when $\kappa=0$ is discussed here since this restriction can be relaxed with little 
extra effort.

Consider the shallow-water equation (1.1) posed in a quarter plane, $x \geq 0$. Assume that a strong solution $u(x, \cdot)$ exists in $H^{4}(0, \infty)$, where $H^{n}(0, \infty)$ is the Sobolev space of (real valued) functions $f$ on the positive real line, $\int_{0}^{\infty}\left(\sum_{k=0}^{n}\left|f^{(k)}(x)\right|^{2}\right) d x<\infty$. Suppose the initial data $u(x, 0)=f(x)$ and smooth boundary data at $x=0, u(0, t)=g(t)$, are given, and these two conditions are compatible at the origin, $g(0)=f(0)$. One can show, by contradiction, that if $g(t) \leq 0$ the (strong) solution is unique for all times of existence [7]. Suppose in fact that there exist two (strong) solutions $u_{1}(x, t)$ and $u_{2}(x, t)$ for the same data, together with their auxiliary fields $m_{1}(x, t)$ and $m_{2}(x, t)$, respectively. The evolution equation for the difference variables $u(x, t)=u_{1}(x, t)-u_{2}(x, t)$, $m(x, t)=m_{1}(x, t)-m_{2}(x, t)$ is

$$
m_{t}=-2\left(m \bar{u}_{x}+\bar{m} u_{x}\right)-\left(u \bar{m}_{x}+\bar{u} m_{x}\right)
$$

where $\bar{u} \equiv\left(u_{1}+u_{2}\right) / 2$ (and $\left.\bar{m} \equiv\left(m_{1}+m_{2}\right) / 2\right)$ denotes the algebraic mean of the two different solutions. Thus, the linearized form of equation (2.6) around the algebraic mean of the hypothetical multiple solutions, equation (6.1), governs the evolution of $u(x, t)$ with homogeneous initial and boundary data $u(x, 0)=0$ and $u(0, t)=0$. Estimates on the evolution of the $H^{1}$-norm $\|u\|_{1}$ can be obtained by multiplying $(6.1)$ by $u(x, t)$ and integrating both sides of the equation. After integration by parts and taking into account the homogeneous boundary condition $u(0, t)=0$, one gets

$$
\partial_{t}\|u\|_{1}^{2}=-\int_{0}^{\infty}\left(3 \bar{u}_{y} u^{2}+\bar{u}_{y} u_{y}^{2}-\bar{u}_{y y y} u^{2}\right) d y+\bar{u}(0, t) u_{x}^{2}(0, t) .
$$

The integral term can be easily bounded by the $H^{1}$-norm and the sup-norm on $\bar{u}_{x}$ and $\bar{u}_{x x x}$ (or $\bar{m}_{x}$, all bounded by the $H^{4}$-norm we assumed to exist), so that (recall $\bar{u}(0, t)=g(t)$ )

$$
\|u(\cdot, t)\|_{1}^{2} \leq C \int_{0}^{t}\|u(\cdot, s)\|_{1}^{2} d s+\int_{0}^{t} g(s) u_{x}^{2}(0, s) d s,
$$

where the constant $C$ is

$$
C=\sup _{s \in[0, t]}\left\{3\left|\bar{u}_{x}\right|_{\infty}(s),\left|\bar{u}_{x x x}\right|_{\infty}(s)\right\} .
$$

The case $g(t)$ non-positive is manifestly handled with inequality (6.3), since the last (inhomogeneous) term generated by the boundary condition can be dropped if $g(t) \leq 0$ while maintaining the inequality. Applying Gronwall's lemma, and taking into account the initial condition $u(x, 0)=0$, shows that $\|u(\cdot, t)\|_{1}=0$, which contradicts the assumption of existence of two distinct (strong) solutions $u_{1}$ and $u_{2}$.

\subsection{Numerical experiments}

For the initial-boundary value problem, an equivalent formulation to (2.2) for the particle method is

$$
\begin{aligned}
& q_{t}(\xi, t)=-\kappa+\frac{1}{2} \int_{\Xi}^{\infty}\left(e^{-|q(\xi, t)-q(\eta, t)|}-e^{-(q(\xi, t)+q(\eta, t))}\right) p(\eta, t) d \eta, \\
& p_{t}(\xi, t)=\frac{1}{2} p(\xi, t) \int_{\Xi}^{\infty}\left(\operatorname{sgn}(\xi-\eta) e^{-|q(\xi, t)-q(\eta, t)|}-e^{-(q(\xi, t)+q(\eta, t))}\right) p(\eta, t) d \eta,
\end{aligned}
$$

where the location $\Xi(t)$ is defined by $q(\Xi(t), t)=0$, and

$$
\frac{1}{2}\left(e^{-|x-y|}-e^{-x-y}\right) \equiv G_{0}(x, y)
$$




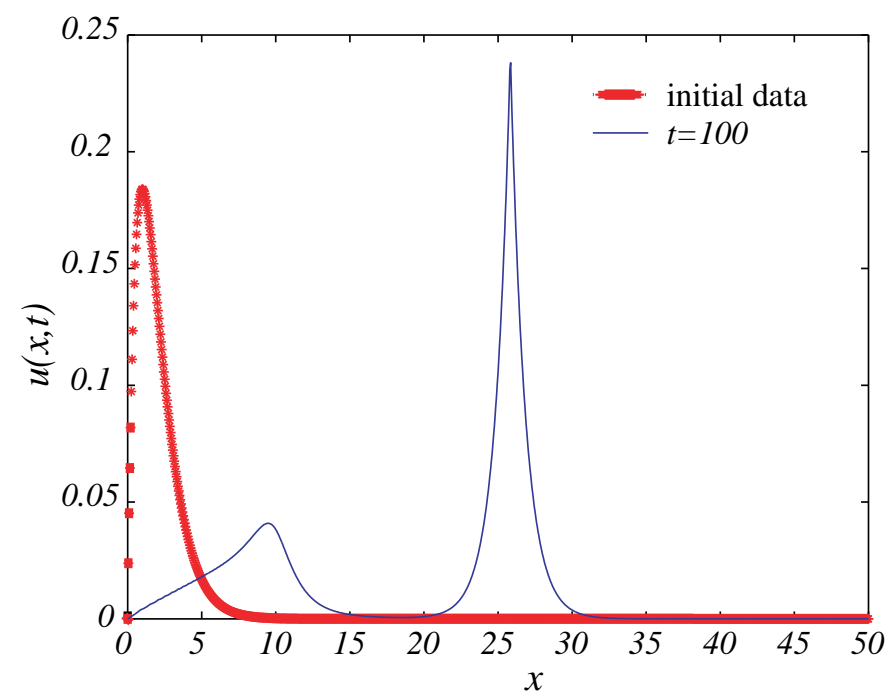

Figure 6.1. A snapshot of numerical solutions of the particle method for the initial-boundary value problem (6.4), with $g(t)=0, \kappa=0, u(x, 0)=x e^{-x} / 2$, with $N=1000$ and grid size $h=0.05$, at time $t=100$.

is the domain Green's function with homogeneous Dirichlet boundary condition. For the special case $g(t) \equiv 0$, we have $\frac{d \Xi}{d t}=0$ and hence $\Xi=0$ at all time. For the numerical example that illustrates our analysis for the initial boundary value problem of the nonlinear shallow-water equation (1.1), we take the initial condition $u(x, 0)=f(x)=x e^{-x} / 2$ (so that $m_{0}(x)=e^{-x}$ ) and homogeneous boundary condition $g(t)=0$ at $x=0$. A snapshot of the solution after some time is shown in Figure 6.1. The grid-refinement study is documented in Table 6.1 for the case $\kappa \neq 0$, where at a fixed time, the difference between solutions obtained by using grid size $h$ and that obtained by $2 h$ decreases by a factor of $1 / 4$, as we refine the mesh by a half grid size. Such a reduction of errors gives evidence to the convergence of the numerical solutions.

\subsection{Lax-pair for homogeneous boundary conditions}

The quarter plane problem for equation (1.1) with homogeneous boundary conditions $u(0, t)=0$, $u(x, t)$ rapidly decaying to zero as $x \rightarrow \infty$, follows again from an isospectral problem. It can be shown that in this case the spectrum of the matrix problem

$$
\lambda\left(\begin{array}{c}
\phi_{1}(\xi, t) \\
\phi_{2}(\xi, t)
\end{array}\right)=\int_{0}^{\infty}\left(\begin{array}{cc}
0 & k(\xi, \eta ; t)+k_{+}(\xi, \eta ; t) \\
k(\xi, \eta ; t)-k_{+}(\xi, \eta ; t) & 0
\end{array}\right)\left(\begin{array}{c}
\phi_{1}(\eta, t) \\
\phi_{2}(\eta, t)
\end{array}\right) d \eta,
$$

\begin{tabular}{|c|c|c|c|c|c|}
\hline$h$ & 0.0125 & 0.00625 & 0.003125 & 0.0015625 & 0.00078125 \\
\hline$\left\|u_{h}-u_{2 h}\right\|_{\infty}$ & $2.52 \mathrm{e}-5$ & $6.77 \mathrm{e}-6$ & $1.68 \mathrm{e}-6$ & $4.18 \mathrm{e}-7$ & $1.05 \mathrm{e}-7$ \\
\hline ratio & & 3.72 & 4.03 & 4.02 & 3.98 \\
\hline$\left\|u_{h}-u_{2 h}\right\|_{2}$ & $4.19 \mathrm{e}-6$ & $1.16 \mathrm{e}-6$ & $2.66 \mathrm{e}-7$ & $6.72 \mathrm{e}-8$ & $1.69 \mathrm{e}-8$ \\
\hline ratio & & 3.64 & 4.32 & 3.96 & 3.98 \\
\hline
\end{tabular}

Table 6.1. The grid-refinement study on the second-order particle method in the infinity and $l_{2}$ norms, respectively, for the initial-boundary value problem (6.4), with $g(t)=0, \kappa=1, u(x, 0)=x e^{-x} / 2$. The computational domain is $[0,50]$ with a uniform grid of size $h$, and the final time is $t=1$. Decreasing the grid size from $h$ to $h / 2$ decreases the difference between the solutions of the two grids by a factor of $1 / 4$. 


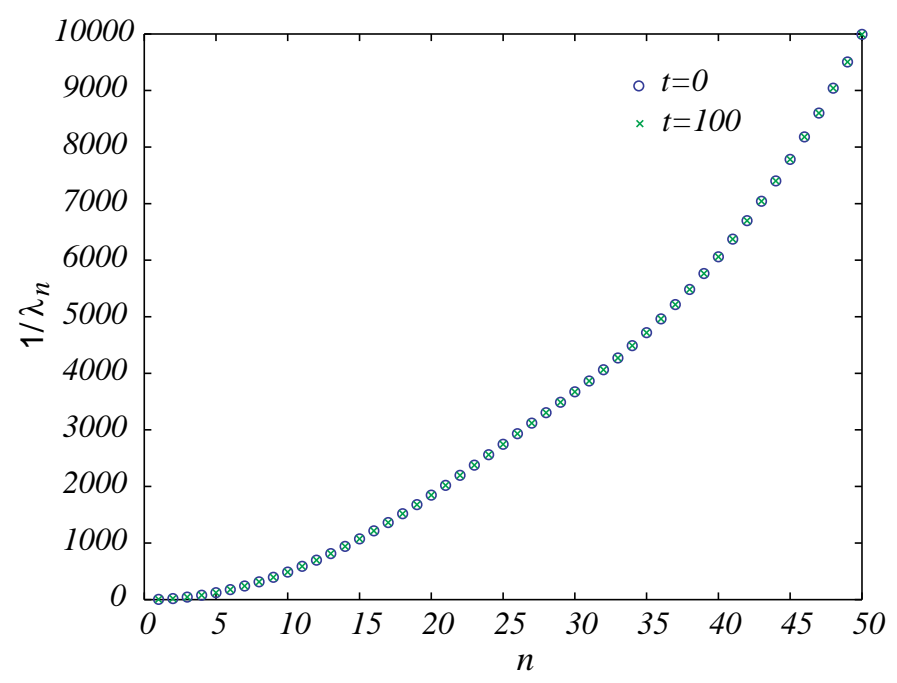

Figure 6.2. Spectrum (first 50 eigenvalues) of the Riemann sums approximation of the eigenvalue problem (6.6) at time $t=0$ (circles) and $t=100$ (crosses), for the initial condition $f(x)=x e^{-x} / 2$, with $\kappa=0$. For this simulation $N=1000, h=0.05$ and $\Delta t=0.025$.

where $k(\xi, \eta ; t)$ is the kernel $(3.3)$ and $k_{+}(\xi, \eta ; t)$ is defined by

$$
k_{+}(\xi, \eta ; t)=\frac{1}{2} e^{-\frac{1}{2}(q(\xi, t)+q(\eta, t))} \sqrt{p(\xi, t) p(\eta, t)},
$$

is invariant in time. Just as in the case of the real line problem, this provides an opportunity to test the numerical scheme by monitoring the spectrum generated by the kernels (3.3) and (6.7) in time. Figure 6.2 shows the evolution of the first significant eigenvalues from those corresponding to the initial condition $f(x)=x e^{-x} / 2$, with $\kappa=0$. As one can see, this part of the spectrum is well preserved by our numerical scheme.

\section{Conclusions}

Our study shows that the particle method can be used as the starting point for implementing an efficient algorithm for numerical solutions of the shallow water equation (1.1). Being completely integrable, the particle method is also a natural choice for this class of integrable nonlinear equations. However, in this respect we notice that complete integrability plays only a marginal role in our implementation, as we used a simple ODE solver to advance the initial data. The solution formulae $[1,5,6]$ for a large number of particles seem to require a numerical approach, which in fact can be more involved than an ODE system solver. This is because of the need to compute determinants of matrices that can be fairly ill-conditioned at sufficiently large times. This issue deserves a more in-depth study, however, since there might be advantages to proceeding from the solution formula. For instance, global accuracy estimates of the algorithm could be established using this formula, in contrast to the simple local accuracy of ODE integrators.

Equations (3.1) have some interest of their own as an example of a completely integrable particle system. Some remarkable generalizations of this system were found some time ago by Professor Calogero in his studies devoted to extending the class of completely integrable equations $[2,3,4]$. As usual, his approach has inspiring consequences. While some of these might not be grounded (yet) 
on physical phenomena, it would be mathematically interesting to follow Calogero's generalizations back to the level of PDE's (as reference [13] demonstrates in a particular case). Results in this direction will be reported in future publications.

Finally, we stress that the particle method is applicable to any equation admitting the same basic integral formulation, regardless of complete integrability. Thus, extensions to other nonintegrable equations with similar structure and, in particular, to more than one spatial dimension are possible and are planned for future work.

\section{Acknowledgments}

RC gratefully acknowledges support from NSF through Grant DMS-0104329. JH thanks NSF support by Grant DMS-0327896. LL is grateful to the Department of Mathematics at the University of North Carolina at Chapel Hill for their support through a postdoctoral fellowship.

\section{References}

[1] R. Beals, D. Sattinger, and J. Szmigielski. Multi-peakons and a theorem of Stieltjes. Inverse Problems 15:L1-L4 1999.

[2] F. Calogero. A solvable Hamiltonian system. J. Math. Phys., 36:4832-4840, 1995.

[3] F. Calogero and J.-P. Francoise. A completely integrable Hamiltonian system. J. Math. Phys., 37:2863-2871, 1996.

[4] F. Calogero. Classical many-body problems amenable to exact treatments. Lecture Notes in Physics Monographs, 66, SpringerVerlag, Berlin 2001.

[5] R. Camassa. Characteristic variables for a completely integrable shallow water equation. In: Boiti, M. et al. (eds.) Nonlinearity, Integrability and All That: Twenty Years After NEEDS 'ry World Scientific, Singapore 2000.

[6] R. Camassa. Characteristics and the initial value problem of a completely integrable shallow water equation. Discrete Cont. Dyn.-B, 3:115-139, 2003.

[7] R. Camassa, J. Huang, and L. Lee. Integral and integrable algorithm for a nonlinear shallowwater wave equation. Submitted, 2004.

[8] R. Camassa and A.I. Zenchuck. On the initial value problem for a completely integrable shallow water wave equation. Phys. Lett. A, 281:26-33, 2001.

[9] R. Camassa, D. D. Holm, and J. M. Hyman. A new integrable shallow water equation. Advances in Applied Mathematics, 31:23-40, 1994.

[10] A. Chertock and D. Levy. Particle methods for dispersive equations. J. Comput. Phys., 171:708-730, 2001.

[11] A. Constantin and H.P. McKean. A shallow water equation on the circle. Commun. Pure Appl. Math., 52:949-982, 1999.

[12] A. Constantin. On the scattering problem for the Camassa-Holm equation. Proc. R. Soc. Lond. Ser. A, 457:953-970, 2001. 
[13] J.K. Hunter and Y.X. Zheng. On a completely integrable nonlinear hyperbolic variational equation. Physica D, 79:361-386, 1994.

[14] R.S. Johnson. Camassa-Holm, Korteweg-de Vries and related models for water waves. J. Fluid Mech., 455:63-82, 2002.

[15] Y. Kodama and K.T-R McLaughlin. Explicit integration of the full symmetric Toda hierarchy and the sorting property. Lett. Math. Phys., 37:37-47, 1996.

[16] J. Moser. Finitely many mass points on the line interacting under the influence of an exponential potential - an integrable system, Lecture Notes in Physics, 38:467-497, 1975.

[17] O. Ragnisco and M. Bruschi. Peakons, r-matrix and Toda lattice. Physica A, 228:150-159, 1996. 